\title{
Nuevo miliario de Augusto procedente de Fuenteguinaldo (Salamanca)*
}

\section{A new Augustus milestone from Fuenteguinaldo (Salamanca)}

\author{
Manuel Salinas de Frías \\ Universidad de Salamanca \\ Juan José Palao Vicente \\ Universidad de Salamanca
}

\begin{abstract}
RESUMEN
Damos a conocer un nuevo miliario del emperador Augusto hallado en el término municipal de Fuenteguinaldo, en el suroeste de la provincia de Salamanca.
\end{abstract}

\section{SUMMARY}

We present a new milestone of Augustus found in Fuenteguinaldo, in the southwest province of Salamanca.

PALABRAS CLAVE: Epigrafía, vías romanas, Lusitania.

KEY WORDS: Epigraphy, Roman roads, Lusitania.

En el transcurso de las labores de restauración de un viejo molino situado en la confluencia del regato Rolloso con el río Águeda, conocido como "Molino del Sobrao", situado en el término municipal de Fuenteguinaldo (Salamanca), se identificaron una serie de elementos de carácter arqueológico reutilizados en la fábrica del edificio. Entre dichos elementos se encontraban el miliario aquí estudiado, un sarcófago antropomorfo, así como diversos elementos arquitectónicos (basas, sillares, cornisas) procedentes del castro de Irueña, que se sitúa sobre el mencionado molino, en la cumbre de la elevación que delimitan ambos, río y arroyo. En el mes de marzo de 2010 esos elementos fueron recuperados y trasladados a las dependencias que la Confederación Hidrográfica del Duero tiene en la presa de Irueña. En la actualidad el miliario se encuentra depositado en el lado oeste del patio del Museo de Salamanca, donde entró el 21 de febrero de

* Este trabajo se ha realizado en el marco del Proyecto de Investigación de la Junta de Castilla y León SA003A10-1 y del Proyecto de Investigación del Micinn HAR 2011-27719.
2010 y donde lo hemos estudiado ${ }^{1}$. Del miliario y del sepulcro antropomorfo da noticia D. Manuel Gómez Moreno en el Catálogo monumental de España, provincia de Salamanca, quien debió verlos durante los trabajos de documentación del mismo, entre 1901 y 1902, ya que dice, refiriéndose a Irueña: “Cerca de la puerta del Sol se han hallado también una caja de piedra, como ataúd, y una estela, arqueada por arriba y con epígrafe, que se llevaron al susodicho molino, y fueron desechas". (Gómez Moreno 2003: 36)². La descripción de la estela a que se refiere, como se puede comprobar por las fotografías que adjuntamos, coincide con la de nuestro miliario, y probablemente Gómez Moreno se refería a él. Es interesante comprobar, en todo caso, que ambos objetos no fueron destruidos como dice, sino que, en realidad, se reutilizaron en la fábrica del molino, empleándose el miliario, concretamente, como dintel de la puerta (Fig. 1).

La pieza está realizada en granito claro. Tiene forma prismática y es más ancha y gruesa en la cabecera que en la base, teniendo aquélla forma ligeramente redondeada. Todo parece indicar que se encuentra completa, ya que no presenta ninguna fractura y conserva incluso la parte final con la que iba hincada en la tierra (Fig. 2).

Tanto la morfología como la ubicación del numeral correspondiente a las millas habituales al inicio del texto no se ajustan a las características de los mi-

\footnotetext{
${ }^{1}$ Desde aquí queremos agradecer la ayuda y facilidades dadas por D. Alberto Bescós, Director del Museo de Salamanca, para el estudio de la pieza y a D. Ángel Macarro Alcalde, Arqueólogo Territorial, por habernos facilitado la información relativa a las circunstancias del hallazgo, así como la foto $n .^{\circ} 1$.

${ }^{2}$ Sobre las fechas de redacción del manuscrito, $c f$. el estudio introductorio de J. R. Nieto en Gómez Moreno 2003: 26.
} 


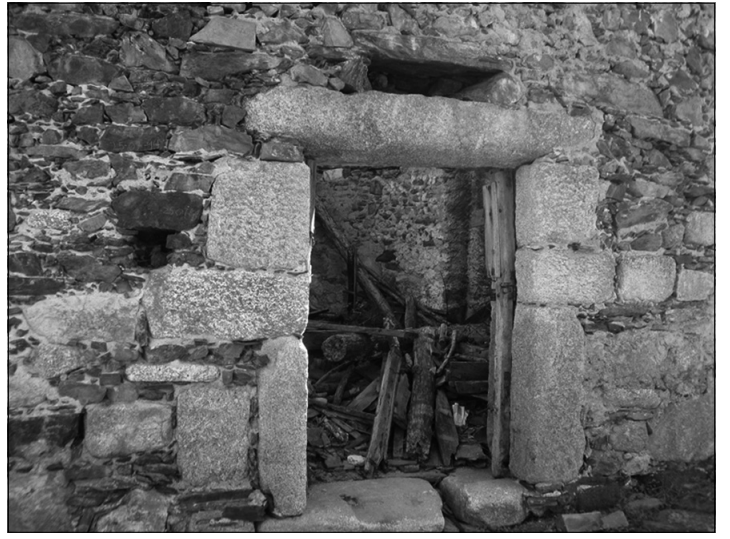

Figura. 1. Emplazamiento original del miliario en el «Molino del Sobrao».

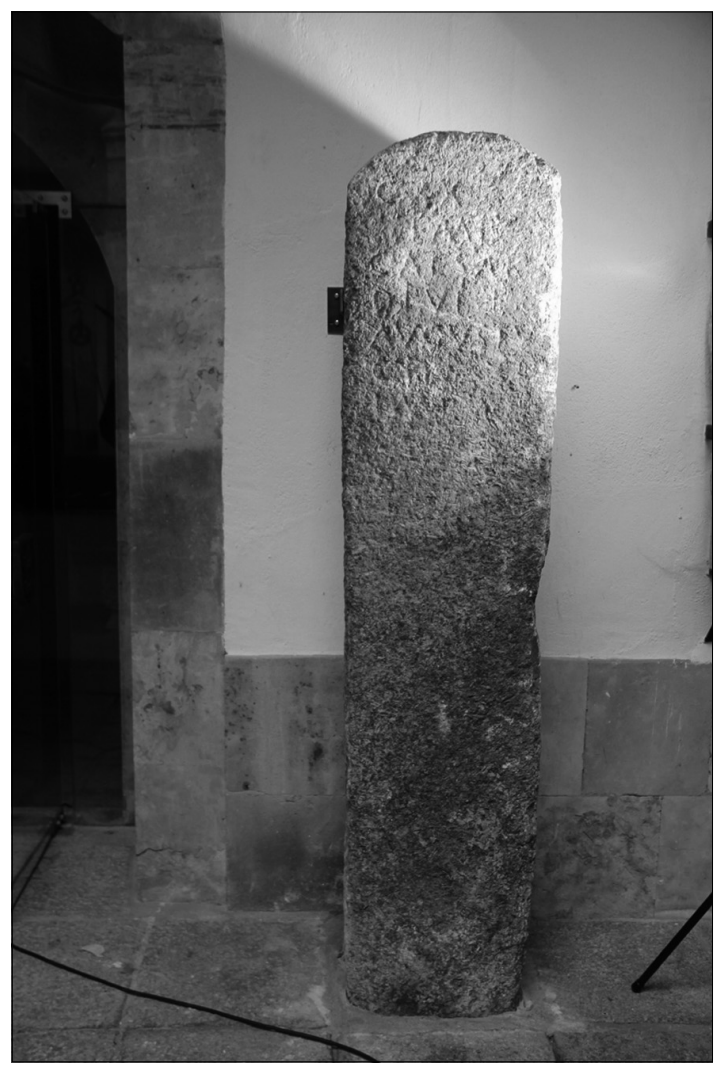

Figura. 2. Vista general del miliario depositado en el Museo de Salamanca.

liarios del período imperial, aunque todos los elementos, tanto formales como textuales, nos inducen a considerarlo un miliario. El texto se desarrolla en la parte superior de la pieza, sin ningún elemento diferenciador con respecto al resto. La superficie está muy ero- sionada, a juzgar por lo superficial de la incisión de las letras, circunstancia que parece tener su origen en el reempleo del ejemplar como material de construcción. Las dimensiones son las siguientes:

Alt. Original: $238 \mathrm{~cm}$. La pieza se encuentra clavada en el suelo del patio del Museo de Salamanca, lo que ha hecho que la parte visible en la actualidad sea de $205 \mathrm{~cm}$.

Anchura máx. $47 \mathrm{~cm}$, anchura mín. $43 \mathrm{~cm}$, Grosor máx: $37 \mathrm{~cm}$, Grosor mín.: $31 \mathrm{~cm}$.

ALTURA LETRAS: Lín. 1: 4,5 cm, Líns. 2-7: $5 \mathrm{~cm}$.

TEXTO (Fig. 3):

CXX
IMP
CAESAR
DIVI F
AVGVSTVS
COS XI
IMP X

LECTURA: CXX / Imp(erator) / Caesar / divi f(ilius) / ${ }^{5}$ Augustus / co(n)s(ul) XI / Imp(erator) X.

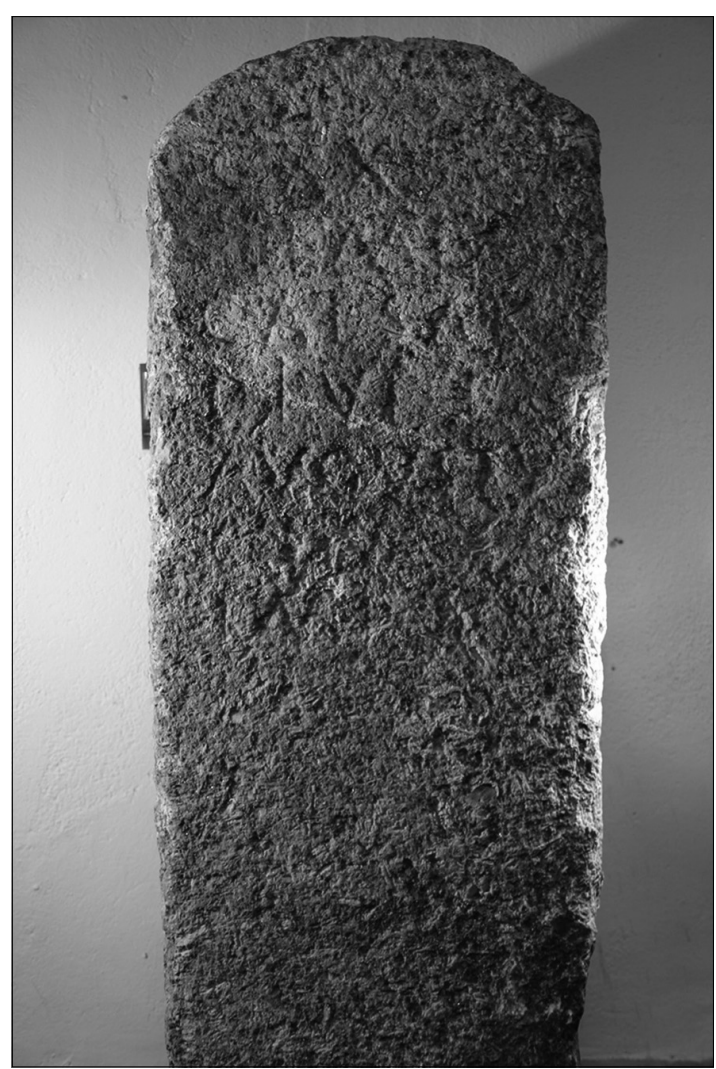

Figura. 3. Detalle del texto (1). 
Conocemos otros miliarios que tienen las mismas características formales que el de Fuenteguinaldo y que permiten afirmar que nos encontramos ante un objeto de esta naturaleza y no de otro tipo como, por ejemplo, un terminus. El primero de ellos es un miliario procedente de la actual localidad tunecina de Hr el-Henza, cerca de Sidi Ali el-Mediouni, que también tiene forma de estela con cabecera semicircular. ${ }^{3}$ Pertenece al reinado de Augusto, aunque no es posible fecharlo con precisión ya que presenta un texto más simple que el que nos ocupa, pues la titulatura del emperador se reduce a Imp(erator) Caesar Augustus. También presenta esta misma forma otro miliario, en este caso fechado en el reinado de Tiberio, de la vía Tacape-Capsa (cerca de Gafsa, Túnez. CIL, VIII, 10023). Según A. M'Charek este tipo de miliarios son característicos de las regiones interiores de Túnez y presentan la misma morfología que las estelas funerarias de los soldados halladas en Haïdra (M'Charek 1992: 158).

En la península Ibérica contamos con otros dos ejemplos casi idénticos al estudiado, que añaden a las semejanzas formales el hecho de hallarse muy próximos geográficamente. ${ }^{4} \mathrm{El}$ primero de ellos procede de la localidad portuguesa de Alfaiates, situada en línea recta a unos 20 kilómetros al sudoeste de Irueña, y está realizado también en granito, presentando la misma forma de estela con cabecera semicircular y sección prismática, aunque su altura es algo inferior $(200 \mathrm{~cm})$. Ya Leite de Vasconcelos lo consideró un miliario, propuesta seguida, aunque con lecturas diferentes, por Lambrino y, recientemente, por F. Patrício Curado. Alarcâo, por su parte, lo consideraba un terminus y M. Osório lo ha interpretado como una inscripción honorífica (Lambrino 1956: n. $^{\circ} 13$; $A E, 1967,185$; Alarcão 1988: 41; Alarcão 2006: 134-136; Osório 2006, n. ${ }^{\circ} 37$, apud Patricio (en prensa)). La lectura propuesta por F. Patrício Curado es la que sigue: [M(ilia) P(assuum) / C $[X X \ldots X] / \operatorname{Im}[p$ (erator) / Caesa[r] / divi f(ilius) / Augustu(s) / c[o(n)s(ul)] XI / imp(erator) [VIII]. Además de la morfología, lo más interesante de este ejemplar es que el numeral identificado con las millas está ubicado al comienzo del texto, tal y como sucede con nuestro miliario.

${ }^{3} A E, 1992,1775$. Un estudio de este miliario en M'Charek 1992: $157-160$ (con foto).

${ }^{4}$ Queremos agradecer muy expresivamente a don F. Patrício Curado que nos haya facilitado, con extraordinaria generosidad, el original de un trabajo suyo en prensa en $O A r$ queólogo Portugués ("Notas sobre dois marcos miliários prismáticos, de Augusto (23 a.C.) da região da Guarda") en el que proporciona tanto las lecturas de los miliarios de Alfaiates y Argomil como las fotografías de los mismos.
El segundo ejemplar procede de Argomil, en el concelho de Pinhel, al nordeste de Guarda. Presenta también sección prismática y forma de estela con cabecera semicircular, aunque en este caso su altura es inferior al anterior $(164 \mathrm{~cm})$. La lectura propuesta por su primer editor A. Vasco Rodrigues es la siguiente: Imp(erator) / Caesar / divi f(ilius) / Augustu[s] / $\operatorname{co}(n) s(u l) X I / i m p\left(\right.$ erator) XIII. ${ }^{5}$

Por otra parte, puede ser tenido en cuenta también un miliario de Bias do Sul (Moncaparacho, Olhâo), que se conserva en el Museo de Moncaparacho (IRCP, 660), cuyo interés radica en el hecho de que, según su editor, aparece desbastado de manera que se presenta como un paralepípedo. La comprobación efectuada sobre la foto que proporciona no nos permite decidir si dicho ejemplar era originariamente de forma prismática o si, por el contrario, lo que nos parece más probable, era cilíndrico y posteriormente fue desbastado de manera cuadrangular muy tosca.

Esta misma morfología — sección prismática con cabecera semicircular más o menos rebajada- la presentan algunas estelas de granito emeritenses, publicadas por J. Edmonson, ${ }^{6}$ que constituyen su tipo IA, cuya cronología oscila entre finales del siglo I a.C. y los inicios del siglo I d.C., coincidiendo, por tanto, con la de nuestro miliario. Este tipo de epigrafía se vincula a los colonos itálicos llegados en época de Augusto.

Otra particularidad tiene que ver con la titulatura imperial, concretamente con la ausencia de la tribunicia potestas, que tampoco aparece en los miliarios de $\mathrm{Hr}$ el-Henza, Alfayates, Argomil, además de otros dos: uno procedente de Casar de Cáceres ${ }^{7}$ y el otro de la localidad portuguesa de Pastoria (Chaves), ${ }^{8}$ en los cuales se omiten tanto el consulado como la salutación imperatoria. Un repaso a los demás miliarios hispanos del período augústeo muestra cómo en todos ellos aparece la potestad tribunicia. La autopsia de la piedra permite descartar la posibilidad de que se hubiera perdido la parte que contendría la potestad tribunicia por efecto de la erosión y su ausencia, por tanto, debe atribuirse a la variabilidad de los formularios epigráficos en una etapa en que se

\footnotetext{
${ }^{5}$ Rodrigues 1980: 99-100; Vaz 1985: 635-639; Curado (art. cit.) interpreta la lectura en dativo y con la salutación imperatoria VIII: Imp(eratori) / Caesar(i) / divi f(ilio) / August $<u>o / \operatorname{co}(n) s($ uli) XI / imp(eratori) VIII.

${ }^{6}$ Edmondson 2006: 25-30. Especialmente, nos. 8-11 con el texto muy pegado al borde superior.

${ }^{7}$ Paredes 1966. Roldán 1971: 51, n. ${ }^{\circ}$ 25; CPILC, 677. El texto dice: Imp(erator) Caesar / Augustus / LIX.

${ }^{8}$ Pastoria (Chaves). Rodríguez Colmenero 1997: 311-312, n. ${ }^{\circ}$ 388: Imp(erator) Cae[s(ar)] / August[us / a] Brac[ara] m(ilia) p(assum) / LV[.].
} 
estaba constituyendo la titulatura imperial (Beltrán 1996: 72).

Por lo que respecta a la cronología, el consulado XI se extiende desde el año 23 a.C., hasta el 1 de enero del 5 a.C., en que Augusto asumió el consulado XII. Por su parte, la salutación imperatoria $\mathrm{X}$ va desde el 1 de julio del 16 a.C. al 5 de marzo del 12 a.C. (Lassère 2007: 999). No obstante, dado el estado de erosión del texto existe la posibilidad de que la salutación imperatoria no sea la $\mathrm{X}$, ya que hay espacio para suponer una o varias astas verticales a continuación de la X, con lo que podría ser la salutación imperatoria XI (12-11 a.C.), XII (10 a.C.) XIII (9 a.C.) o XIIII (8-6 a.C.). Por tanto, la datación del miliario puede oscilar entre el 1 de julio del año 16 a.C., fecha del inicio de la salutación imperatoria $X$, y el 5 de marzo del año 6 a.C., última fecha en la que coinciden el consulado XI y la salutación imperatoria XIIII.

En Hispania conocemos un total de veintitrés miliarios con el consulado XI que contienen salutaciones imperatorias que comiencen por $\mathrm{X},{ }^{9}$ combinación que se ajusta al texto que aquí presentamos. Esta cifra supone más de la mitad de los miliarios del reinado de Augusto conservados en la península ibérica. ${ }^{10}$ Los más abundantes son los que muestran la combinación del consulado XI y la salutación imperatoria XIIII, que alcanzan más del $80 \%$ del total ${ }^{11}$

\footnotetext{
${ }^{9}$ Sin embargo, no todos los textos conservados contienen ese consulado ni salutación imperatoria. Una parte de ellos están reconstruidos a partir de la potestad tribunicia o en función de la presencia en la misma vía de miliarios con esa titulatura. Así sucede con los ejemplos procedentes de Castiliscar (Lostal 1992, n. ${ }^{\circ}$ 19); Ilche (Lostal 1992: n. ${ }^{\circ}$ 16); Gallur (HEp 5, 1995, 919); Vélez Rubio (CIL, II, 4939); Torrente de Cinca (CIL, II, 4921); Candasnos (CIL, II, 4923); Peñalba (CIL, II, 4917) y El Hito (HEp, 5, 1995, 327). Por el contrario, contamos con ejemplos completos del consulado y / o la salutación imperatoria: Calahorra $(A E, 1997,911)$; Ejea de los Caballeros (Lostal 1992: n. ${ }^{\circ}$ 18); Vilasar de Mar (Lostal 1992: . $^{\circ}$ 7); Barcelona (Lostal 1992: n. ${ }^{\circ}$ 8); Tarragona (CIL, II, 6240); Lorca (CIL, II, 4937); Chirivel (CIL, II, 4938); Baza (AE, 1982, 627); La Guardia (CIL, II, 4931); Totana (CIL, II, 4936); Mazarrón (Lostal 1992: n. $\left.{ }^{\circ} 25\right)$; Torrente de Cinca (CIL, II, 4920; CIL, II, 4922); Cardiel (HEp, 5, 1995, 360); Padilla de Abajo (Lostal 1992: n. ${ }^{\circ}$ 21); Castiliscar (Lostal 1992: n. ${ }^{\circ}$ 20).

${ }^{10}$ Hasta la fecha hay identificados cuarenta y siete, aunque la adscripción de algunos de ellos al reinado de Augusto no tiene como base el texto sino datos indirectos. Sobre el número de miliarios vid. Solana, Sagredo 2008: 125.

${ }^{11}$ En total 20 de esos 23. De ellos, ocho están reconstruidos a partir de la tribunicia potestad o de otros datos indirectos: Ilche (Lostal 1992: n. ${ }^{\circ}$ 16); Gallur (HEp, 5, 1995, 919); Vélez Rubio (CIL, II, 4939); Torrente de Cinca (CIL, II, 4921); Candasnos (CIL, II, 4923); Peñalba (CIL, II, 4917) у El Hito (HEp, 5, 1995, 327). Con el consulado XI y la salutación imperatoria XIII hay tres: Calahorra $(A E, 1997,911)$; Ejea de los Caballeros (Lostal 1992: n. ${ }^{\circ}$ 18) y Castiliscar
}

y que proporcionan la fecha 8-6 a.C. Esto podría ser argumento para pensar en una fecha cercana a esta última para nuestro miliario, aunque la distancia geográfica que existe con respecto a aquellos induce a ser muy cautos a la hora de establecer una cronología.

En lo que respecta a Lusitania, eran conocidos hasta ahora otros tres miliarios de Augusto. El primero de ellos es con seguridad un falso, conocido solamente por traducción manuscrita, cuyo estilo florido claramente evidencia que es una fabulación erudita (CIL, II, 443*; Roldán 1971: 65, n. ${ }^{\circ}$ 19; Salinas 2007: 20). El segundo es una pieza hallada en 1966 en la localidad cacereña de Casar de Cáceres, citada anteriormente, que se encuentra desaparecida en la actualidad. Como hemos dicho, presenta un texto poco habitual, ya que solo aparece el nombre del emperador y las millas. El tercero es el miliario de Bias do Sul (Moncaparacho, Olhâo), ya citado, probablemente perteneciente a la vía de Balsa a Ossonoba, aunque lo fragmentario de su texto hace que la atribución a Augusto no deje de ser hipotética (IRCP, 660).

El miliario que damos a conocer presenta por tanto distintos motivos de interés: por un lado, su situación geográfica atestigua una vía diferente de la de Emerita a Asturica y, por otro, añade un testimonio nuevo de la actividad constructiva romana en el norte de Lusitania, una zona donde no se conocían intervenciones viarias hasta la época de Nerón. A este interés primario, se suma el hecho de constituir un nuevo dato histórico, a añadir a los escasísimos que se poseían de la actividad de Augusto en esta zona, que hasta el presente se limitaban a los termini datables entre los años 4-6 d.C. que atestiguan una reorganización territorial profunda de Lusitania septentrional (Ariño 2005: 95-112).

Desconocemos la vía a la que podría pertenecer este miliario, pero, dada su situación geográfica, hay que descartar que perteneciera a la denominada Vía de la Plata, que discurría mucho más hacia el este. J. M. Fernández Corrales supone una vía de Turmuli (Alconétar) al puerto de Perales por Caurium que alcanzaría Ciudad Rodrigo, de la que no quedaría vestigio arqueológico alguno pero cuya existencia hay que suponer para unir una serie de puntos estratégicos (Fernández Corrales 1987: 85-86). Esta vía discurriría por Garrovillas, Portezuelo, Torrejoncillo, Coria, Calzadilla, Moraleja y Perales del Puerto, entre Acebo y Gata. Ciertamente, una vez superado el

(Lostal 1992: n. ${ }^{\text {19 }}$ ), y sólo uno con el consulado XII y la salutación imperatoria XIV: Castiliscar (Lostal 1992: n. ${ }^{\circ} 20$ ). 
Puerto de Perales, esta vía discurriría cerca de Fuenteguinaldo, en cuyo término se ha hallado el miliario. Pero M. García de Figuerola supone también otra vía romana que ascendería desde Extremadura hasta Salamanca por el Puerto de Santa Clara, desde San Martín de Trevejo hasta Fuenteguinaldo (García de Figuerola 1999: 116-119), precisamente, enlazando la cabecera del río Eljas con la cabecera del Águeda, junto a cuyo curso se ha encontrado el miliario. Es posible por tanto que éste perteneciera a esta segunda vía o, mejor dicho, que la vía romana no discurriera por donde la principal carretera moderna, la del Puerto de Perales, sino por el antiguo camino de Santa Clara, muy frecuentado hasta mediados del siglo xx por caballerías y jornaleros extremeños que iban a segar a Castilla. CXX millas dan una distancia aproximada de $180 \mathrm{kms}$ a contar desde Mérida. La distancia actual por carretera, usando vías rápidas según la versión informática de la Guía Michelín, es de $204 \mathrm{kms}$. Puesto que evidentemente el trazado antiguo debía diferir en algo del moderno; y el actual, además, rodea por el Puerto de Perales, es posible que el miliario corresponda a una vía que se iniciaba en Emerita y se dirigía a algún punto del occidente salmantino o de los territorios vecinos de Portugal, en todos los cuales existen zonas mineras explotadas por los romanos.

Otro elemento que hay que valorar es la naturaleza jurídico-política del castro de Irueña, en las inmediaciones de una de cuyas puertas se emplazaba el miliario según Gómez Moreno, que debió ser, por tanto, una mansio de esta vía (De Francisco 1989; Mangas 1992: 258, 262-263; Salinas de Frías 2008: 347-348) (Fig. 4).

La cuestión fue planteada por J. Mangas sobre la base de dos inscripciones, una de ellas desaparecida, que Hübner cita como procedentes de Ciudad Rodrigo pero que Fita atribuye a Irueña. ${ }^{12}$ Una de ellas es un pedestal de estatua dedicado a Pértinax por el $o($ rdo) M(unicipii) U(runiensis) y la otra es una dedicatoria a Domiciano, hecha $D$ (ecreto) $D($ ecurionum), pero de las que Martín Valls afirma taxativamente que ambas se encontraron al abrir las zanjas de un edificio de Ciudad Rodrigo (Martín Valls, R. 1976: 387; Fernández Guerra 1899: 105). Para Mangas, si Fita estuviera en lo cierto, es decir, que

${ }^{12}$ CIL, II, 863: Imp(eratori) Caes(ari) / L(ucio) Sept(imio) Severo / Pertinaci Aug(usto) / o(rdo) M(unicipii) U(runiensis) ex / A. P. V.; CIL, II, 862: Imp(eratori) Caes(ari) divi / Vespasiani f(ilio) Domitiano Aug(usto) / pont(ifici) max(imo) trib(unicia) / pot(estate) imp(eratori) II p(atri) p(atriae) co(n)s(uli) / VIII desig(nato) VIIII / d(ecreto) d(ecurionum); Fita 1912: 446 ss.; citadas por Mangas 1992: 262.

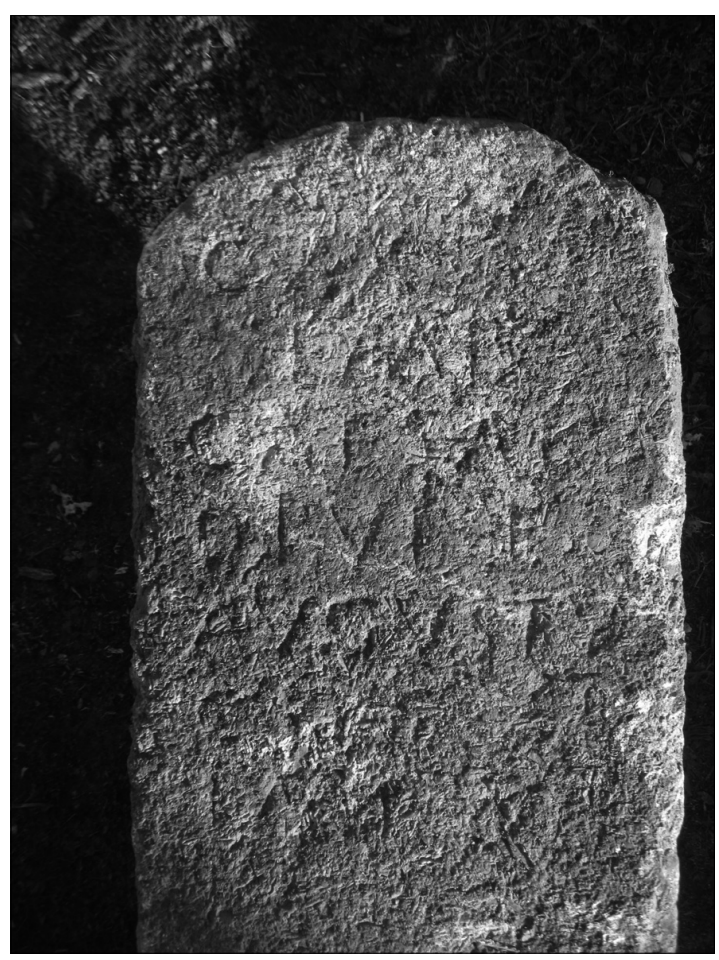

Figura. 4. Detalle del texto (2).

ambas inscripciones fuesen del castro de Irueña, esto probaría su naturaleza de municipio Flavio; por el contrario, si se acepta lo que dice Martín Valls, habría que aceptar "que el municipio Flavio que hizo estas dedicaciones estaba en el castro romanizado de Ciudad Rodrigo, lo que nos llevaría, fácilmente, a aceptar la igualdad Mirobriga=Ciudad Rodrigo" (Mangas 1992: 263), existiendo un error de lectura en la primera de las inscripciones, que no diría $o(r d o)$. M(unicipii) U(runiensis), sino o(rdo) Mir(obrigensis), siendo el castro de Irueña un núcleo situado dentro del territorium de Mirobriga, pero no el centro de otra civitas. La confusión acerca de la procedencia exacta de distintos epígrafes se ve alimentada por el hecho de que algunos autores han supuesto que, con el aumento de importancia de Ciudad Rodrigo a partir del siglo XII, muchos materiales de Irueña pudieron ser movidos desde aquí a allá, sin que tampoco exista prueba cierta o documental de dichos traslados de material. Entre otros se han citado los dos termini augustales (CIL, II, 857 y 858) así como las tres columnas que blasonan el escudo de la ciudad. Mangas dice que en 1972, cuando las columnas estaban desmontadas para ser trasladadas junto a la entrada de la población por la carretera de Salamanca, pudo medirlas y su diámetro corresponde al de las basas 


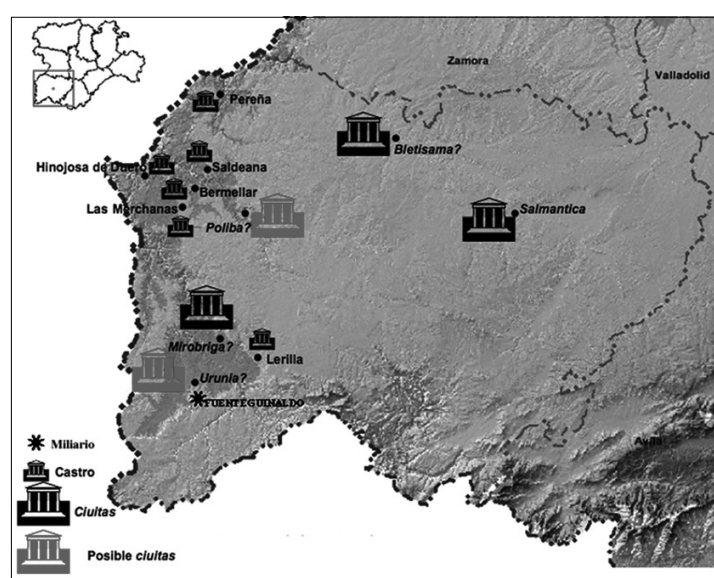

Figura. 5. Mapa de la provincia de Salamanca con la localización de Fuenteginaldo.

de un templo romano cuyos restos pueden verse en Irueña (Mangas 1992: 257), que tal vez sea al que pertenecieran las tres columnas que vio Sánchez Cabañas, de quien recoge el dato Gómez Moreno pensando que unas y otras tal vez fueran las mismas y que desde Irueña se llevasen a Ciudad Rodrigo en el siglo XVI. ${ }^{13}$ Sin embargo, parece existir constancia documental de que las columnas de Ciudad Rodrigo aparecieron en el interior de la población, cerca de las llamadas "Carnicerías Viejas" (Martín Valls 1965: 71-98), estando además la innegable constancia arqueológica de la existencia de un núcleo de población de la Segunda Edad del Hierro bajo el solar intramuros de Ciudad Rodrigo que, a partir de época de Claudio, experimenta una monumentalización compatible con su consideración como una civitas romana. Esta situación parece reflejar más bien la existencia de dos civitates próximas, Urunia y $\mathrm{Mi}$ robriga, para cuyas obras públicas se utilizaron módulos semejantes, tal vez porque se abastecían de un mismo taller comarcal. ${ }^{14} \mathrm{~A}$ veces se ha argumentado la proximidad geográfica entre ambas poblaciones para excluir que fuesen dos civitates distintas y suponer que, forzosamente, una debiera estar incluida en el territorium de la otra. Sin embargo, dentro de la misma provincia de Salamanca, tenemos dos poblaciones de las que nos consta que en la Antigüedad fueron civitates distintas: Salmantica (Salamanca) y Bletisama (Ledesma). La distancia entre ambas es

${ }^{13}$ Gómez Moreno 2003: 35 citando a Sánchez Cabañas. Sospecha que sean las mismas que las de Ciudad Rodrigo, trasladadas a ese lugar.

${ }^{14}$ Salinas 2008: 343, a favor de la identificación de Ciudad Rodrigo con Mirobriga y la consideración de Irueña/Urunia como un municipio independiente. de unos 29 kilómetros. La distancia entre Ciudad Rodrigo e Irueña de unos 28 kilómetros. Por tanto, y con independencia de su estatuto jurídico, si ambas o solo una de las dos fueron municipios, nada impide que ambas poblaciones fueran civitates diferentes, unidas tal vez por la misma vía, de la cual el miliario que presentamos es el primer testimonio conocido (Fig. 5).

\section{BIBLIOGRAFÍA}

Alarcão, J. de 1988: O Domínio Romano em Portugal, Lisboa.

Alarcão, J. de 2006: "Notas de arqueologia, epigrafia e toponímia", Revista Portuguesa de Arqueología 1, 131-147.

Ariño Gil, E. 2005: "La Hispania Citerior occidental y la Lusitania septentrional entre Augusto y los Flavios: el ager per extremitatem mensura comprehensus", L'Aquitaine et l'Hispanie septentrionale à l'époque julio-claudienne. Organisation et exploitation des espaces provinciaux. Colloque Aquitania, Bordeaux, 95-112.

Beltrán Lloris, F. 1996: "Un nuevo miliario y una nueva vía augústeos en Jatiel (Teruel)", Kalathos $15,67-78$.

De Francisco, J. 1989: Conquista y romanización de Lusitania, Salamanca.

Edmondson, J. 2006: Granite funerary stelae from Augusta Emerita, Madrid.

Fernández Corrales, J. M. 1987: El trazado de las vías romanas en Extremadura, Cáceres.

Fernández-Guerra, A. 1899: "Piedra romana Terminal de Ledesma", Boletín Real Academia de la Historia 15, 105.

Fita, F. 1912: "La diócesis y el fuero eclesiástico de Ciudad Rodrigo en febrero de 1161", Boletín Real Academia de la Historia 61, 437.

García de Figuerola, M. 1999: Arqueología romana y altomedieval de la Sierra de Gata (El Valle de Valverde. Provincia de Cáceres), Cáceres.

Gómez Moreno, M. 2003: Catálogo monumental de España. Provincia de Salamanca. Reproducción facsímil. Estudio introductorio de J. R. Nieto González, Salamanca.

Lambrino, S. 1956: "Les inscriptions latines inédites du Musée Leite de Vasconcelos”, O Arqueólogo Português 3, 2. a série, 5-73.

Lassère, J. M. 2007: Manuel d'épigraphie romaine, Paris.

Lostal Pros, J. 1992: Los miliarios de la provincia Tarraconense, Zaragoza. 
Mangas, J. 1992: "Ciudades antiguas de la provincia de Salamanca (siglo III a.C.- Diocleciano)", Actas I Congreso Historia de Salamanca, Salamanca, 251-268.

Martín Valls, R. 1965: "Investigaciones arqueológicas en Ciudad Rodrigo", Zephyrus 15, 71-98.

Martín Valls, R. 1976: "Nuevos hallazgos arqueológicos en Ciudad Rodrigo", Zephyrus 26-27, 373-388.

M'Charek, A. 1992: "Un itineraire inédit dans la région de Maktar : tronçon de la voie Augustéenne Carthage-Ammaedara", Bulletin Archéologique Comité Travaux Historiques Scientifiques, $n$.s. Afrique du Nord 22, 153-167.

Osório, M. 2006: O Povoamento Romano do Alto-Côa (tese de mestrado em Arqueologia apresentada à Faculdade de Letras de Universidade de Coimbra em 2000).

Paredes, S. 1966: Diario Extremadura, (15, 1, 1966).

Rodrigues, A. V. 1980: "Ronda do Distrito: registos de Arqueologia”, Altitude 1, 2. ${ }^{\text {a }}$ série, 99-100.

Rodríguez Colmenero, A. 1997: Aquae Flaviae I. Fontes epigráficas da Gallaecia meridional interior, Chaves.

Roldán Hervás, J. M. 1971: Iter ab Emerita Asturicam, Salamanca.

Salinas de Frías, M. 2007: "La historiografía sobre la Vía de la Plata”, G. Gillani y M. Santonja (eds.), Arqueología en la Vía de la Plata (Salamanca), Salamanca, 13-32.

Salinas de Frías, M. 2008: "Problemas de ordenación territorial y social de Salamanca en la Antigüedad", J. Mangas y M. A. Novillo (eds.), El territorio de las ciudades romanas, Madrid, 333358.

Solana Sainz, J. M. y Sagredo San Eustaquio, L. 2008: La política viaria en Hispania. Siglos I-II d.C., Valladolid.

Vaz, J. L. da Inês 1985: "Inscrição romana de Argomil (Pinhel)", Beira Alta 44-4, 635- 639.

Recibido: 18-04-2011

Aceptado: 02-12-2011 